\title{
Chemosensory interaction: acquired olfactory impairment is associated with decreased taste function
}

\author{
Basile N. Landis • Mandy Scheibe $\cdot$ Cornelia Weber • \\ Robert Berger • Annika Brämerson • \\ Mats Bende • Steven Nordin · Thomas Hummel
}

Received: 17 November 2009/Revised: 2 February 2010/Accepted: 17 February 2010/Published online: 11 March 2010

(c) Springer-Verlag 2010

\begin{abstract}
Olfaction, taste and trigeminal function are three distinct modalities. However, in daily life they are often activated concomitantly. In health and disease, it has been shown that in two of these senses, the trigeminal and olfactory senses, modification of one sense leads to changes in the other sense and vice versa. The objective of the study was to investigate whether and (if so) how, the third modality, taste, is influenced by olfactory impairment. We tested 210 subjects with normal $(n=107)$ or impaired $(n=103)$ olfactory function for their taste identification capacities. Validated tests were used for olfactory and gustatory testing (Sniffin' Sticks, Taste Strips). In an additional experiment, healthy volunteers underwent reversible olfactory cleft obstruction to investigate shorttime changes of gustatory function after olfactory alteration. Mean gustatory identification (taste strip score) for the subjects with impaired olfaction was $19.4 \pm 0.6$ points and $22.9 \pm 0.5$ points for those with normal olfactory
\end{abstract}

B. N. Landis $(\bowtie) \cdot$ M. Scheibe $\cdot$ C. Weber $\cdot$ R. Berger .

T. Hummel

Smell and Taste Clinic, Department of Otorhinolaryngology,

University of Dresden Medical School, Technische Universität

Dresden, Fetscherstrasse 74, 01307 Dresden, Germany

e-mail: bnlandis@yahoo.co.uk

\section{B. N. Landis}

Division of Otorhinolaryngology-Head and Neck Surgery, Department of Clinical Neurosciences, Geneva Neuroscience Center, University of Geneva Medical School, Geneva, Switzerland

A. Brämerson · M. Bende

Department of Otorhinolaryngology, Central Hospital, Skövde, Sweden

S. Nordin

Department of Psychology, University of Umea, Umeå, Sweden function $(t=4.6, p<0.001)$. The frequencies of both, smell and taste impairments interacted significantly $\left(\mathrm{Chi}^{2}\right.$, $F=16.4, p<0.001$ ), and olfactory and gustatory function correlated $\left(r_{210}=0.30, p<0.001\right)$. Neither age nor olfactory impairment cause effects interfered with this olfactory-gustatory interaction. In contrast, after shortlasting induced olfactory decrease, gustatory function remained unchanged. The present study suggests that longstanding impaired olfactory function is associated with decreased gustatory function. These findings seem to extend previously described mutual chemosensory interactions also to smell and taste. It further raises the question whether chemical senses in general decrease mutually after acquired damage.

Keywords Taste - Gustation - Olfaction - Interaction · Chemical senses

\section{Introduction}

We sense our chemical environment using three systems: the somatosensory, mediated by the trigeminal nerve, the gustatory and the olfactory. Although these three systems identify and mediate different qualities, daily life activities such as eating, breathing or drinking makes them often work simultaneously. Consequently, olfactory, trigeminal and gustatory fibers are concomitantly activated. At a peripheral level, olfactory and trigeminal fibers are intimately intermingled within the olfactory cleft region [29] and taste and trigeminal fibers are intermingled at the tip of the tongue [35]. In contrast, olfactory and gustatory fibers are not found together at any peripheral site (e.g. tongue or olfactory cleft). On a central nervous level, gustatory, olfactory and trigeminal afferent information converges at 
the orbitofrontal cortex [27]. As a consequence, in healthy subjects, these three modalities frequently interact $[4,7$, $20]$. Hence, loss or alteration of one modality could influence the other chemical senses. This question has been addressed by several authors, who could show that olfactory impairment lowers trigeminal function and, vice versa, trigeminal impairment decreases olfactory function [3, 17]. To date, little is known as to whether and how gustatory function, as a third player within the chemical senses, changes when olfactory function is altered. In agreement with the aforementioned reports' hypotheses that impairment of a chemical sense attenuates the other chemical senses, one would expect gustatory function to decrease if olfaction is impaired. The aim of this study was to investigate gustatory function in subjects with normal or impaired olfactory function. To gain potential insight into the time frame requested for such interactions, an addition experiment was conducted in which the olfactory cleft was temporarily blocked.

\section{Materials and methods}

\section{Participants}

Olfactory and gustatory identification were tested in 210 subjects, of whom 103 (62 women, 41 men, mean age $53.5 \pm 1.4$ years) had impaired olfactory identification scores and 107 (62 women, 45 men, mean age $51.8 \pm 1.3$ years) had normal scores. Impaired olfactory function was due to causes such as head trauma (30\%), upper respiratory tract infection (28\%), chronic rhinosinusitis (12\%), toxic exposure (2\%), congenital (1\%), miscellaneous $(10 \%)$ or unknown reasons $(17 \%)$. All subjects were previously investigated for long-lasting chemosensory disorders at the olfactory outpatient clinic according to the routine workup (for details see [8, 22]). Intake of drugs known to alter gustatory function [9] were exclusion criteria. Since qualitative taste/smell dysfunction (e.g. dysgeusia, phantosmia or parosmia) cannot be assessed reliably and we wanted to investigate only interactions of measurable olfactory and gustatory function, such qualitative distortions were further exclusion criteria. The study was conducted according to the Declaration of Helsinki on Biomedical Research Involving Human Subjects and approved by the institutional review board.

\section{Chemosensory testing}

Olfactory function was tested with the identification kit of the Sniffin' Sticks test battery, consisting of 16 familiar odors. According to previously published [21] and recently updated [18] norm values of this widely used European smell test, more than 11 correctly identified odors were considered consistent with normal olfactory function. Gustatory function was examined by using "taste strips", a clinical identification test based on impregnated filter papers. Taste identification is considered normal when 19 or more of 32 presented taste strips are correctly identified (for details see $[23,25]$ ).

\section{Olfactory cleft blocking experiment}

In an additional experiment, 15 volunteers (6 women, 9 men, mean age $24 \pm 4.1$ years), were tested for gustatory function before and after mechanical obstruction of the olfactory cleft for $1 \mathrm{~h}$ using sponges, leading to transient, reversible lowering of olfactory function (for details see [26]).

\section{Statistics}

Results were analyzed using SPSS 15.0 for Windows ${ }^{\mathrm{TM}}$ (SPSS Inc., Chicago, IL, USA). Descriptive statistics are presented as means and standard errors of the means $( \pm$ SEM). $T$ tests for independent samples were employed for comparisons between groups. Analysis of variance was used to control for the etiologies. Correlation analyses were performed using Spearman statistics and partial correlations controlled for age as confounding factors. Analyses of frequencies were calculated using Chi-Square tests. The alpha level was set at 0.05 .

\section{Results}

The mean olfactory identification score for subjects with impaired olfaction was $7.1 \pm 0.3$ points (highest score 1 ; lowest score 11) and $13.6 \pm 0.1$ points (highest score 16; lowest score 12) for subjects with normal olfactory identification $(t=20.9, p<0.001)$. Mean gustatory identification, expressed as taste strip score, for subjects with impaired olfaction was $19.4 \pm 0.6$ points (highest score 5; lowest score 30) and $22.9 \pm 0.5$ points (highest score 9; lowest score 31) for those with normal olfactory function $(t=4.6, p<0.001$; Fig. 1). There was no significant age difference between the two groups (altered olfaction $53.5 \pm 1.4$ years, normal olfaction $51.8 \pm 1.3$ years; $t=0.8, \quad p=0.39$ ). A significant positive correlation was found between olfactory and gustatory function $\left(r_{210}=0.30, p<0.001\right)$ which persisted after age corrected partial correlation $\left(r_{210}=0.29, p<0.001\right)$. Among subjects with impaired olfaction, 45 had altered gustatory function (less than 19 taste strips identified) and 62 normal taste functions. Only 17 subjects with normal olfactory 


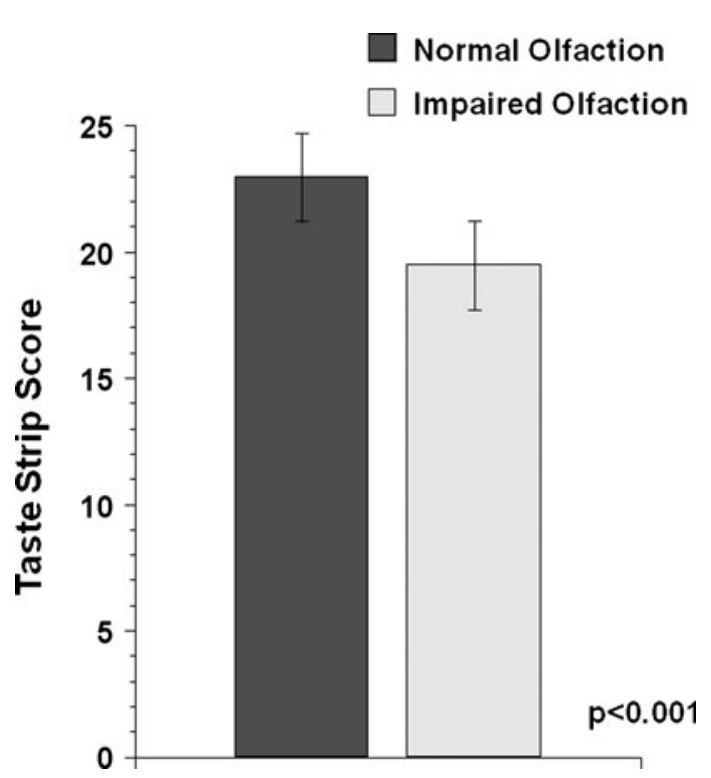

Fig. 1 Mean scores for gustatory function in subjects with normal versus impaired olfactory function. Subjects with impaired olfactory function exhibit decreased taste function compared to subjects with a normal sense of smell

function had altered taste function and 86 had both normal taste and olfactory functions. Analyses of these frequency rates yielded a significant association of impaired olfactory function with decreased gustatory function $\left(\chi^{2}\right.$ test, $F=16.4$, degrees of freedom $(d f)=1 ; \quad p<0.001$; Table 1). Further analyses of variance with a special focus on the causes of impaired olfactory function did not reveal that the association between impaired olfactory and taste functions was restricted to any particular cause $[F(7,36)=1.04, p=0.41]$.

\section{Olfactory cleft blocking experiment}

In the additional experiment, olfactory function was transiently lowered by $30 \%$ (mean olfactory threshold before obstruction: $6.7 \pm 0.3$ points, with obstruction $4.7 \pm 0.3$ points, $p<0.001$ ), whereas gustatory function remained uninfluenced by the olfactory cleft obstruction (mean taste strip score before obstruction: $24.0 \pm 0.9$ points, with obstruction $24.1 \pm 1.6$ points, $p=0.9$ ).

\section{Discussion}

The main finding of the present study is that long-term olfactory impairment lowers gustatory function. Despite its statistical significance, it has to be kept in mind that this decrease in gustatory function is of moderate and subclinical extent and most likely remains unnoticed by subjects with impaired olfactory function. Consequently, studies using very rough taste testing or small sample sizes, might not have found this interaction. Further, this concomitant decrease in taste function seems not to appear after short term olfactory impairment but appears to require longer olfactory impairment to be present. Although age is a known factor in decreased taste function [23], we could reasonably exclude this co-factor as a possible cause of our findings. This effect was further unrelated to the origin of olfactory impairment. Particularly, subjects with posttraumatic olfactory impairment did not contribute more than other investigated subjects to the interaction observed here.

The question of how human sensory function changes after acquired loss or impairment of another sensory system has been addressed numerous times for interactions between sight, audition and tactile function. This literature shows that acquired loss or impairment of one sensory system leads to compensatory mechanisms in other sensory systems due to remarkable cortical plasticity [2, 5, 24, 34]. In analogy to these observations in sight, audition or tactile function, one could speculate that olfactory dysfunction should not lead to decreased taste function. However, this analogy can probably not been drawn that simply, since distinct anatomical and functional similarities exist among the chemical senses, suggesting a higher degree of interdependence than between vision and audition. In contrast to vision or audition the chemosensory organs have to be considered from an anatomical point of view as double sensory organs. The tongue has a dense and overlapping gustatory and trigeminal innervation similar to that found within the olfactory cleft where trigeminal fibers are densely present and even project into the olfactory bulb [27, 29]. Further, all three chemical senses have projections to the orbitofrontal cortex, which is considered the secondary olfactory and gustatory cortex [27]. Within the orbitofrontal cortex, multimodal neurons responding to taste,

Table 1 Frequencies of normal and decreased olfactory and gustatory scores

\begin{tabular}{lll}
\hline & Decreased taste strip score $(<19$ points $)$ & Normal taste strip score $(\geq 19$ points) \\
\hline Impaired olfactory function (identification $<12)$ & 45 & 62 \\
Normal olfactory function (identification $\geq 12)$ & 17 & 86 \\
\hline
\end{tabular}

The $\chi^{2}$ test showed a significant interaction, with more subjects having low taste strip scores in the impaired olfactory group, than could be expected. This suggests a link between impaired olfactory identification score and decreased taste strip values

$\chi^{2}$ test; $F=16.4 ; p<0.001$ 
touch and olfaction have been described [28]. The orbitofrontal cortex seems to play a crucial role within the central processing of chemosensory information [27]. On a daily functional level, eating and drinking always leads to a concomitant co-activation of taste, olfaction and even the trigeminal system. Finally, trigeminal-taste interactions have been reported, suggesting that touch or temperature can elicit gustatory perceptions [6, 32].

For these reasons, one cannot simply state that compensatory mechanisms seen for sight, audition and touch can be translated into chemical senses. Chemical senses might be different when it comes to mutual interaction in health and disease.

The present findings seem to fit the assumption that the three chemical senses are functionally linked, and that change in one sense impacts on the other chemical senses. This functional cross linking is assumed to be due to the repeated co-activation between the three chemosensory systems in everyday life [30]. In patients who suddenly lose one chemical sense (e.g. olfaction), this missing afferent information would also lead to a decreased chemosensory integration with lowered overall performances in the remaining chemical senses (e.g. trigeminal function). Previous studies in patients seem to confirm that $[3,17]$. Very recent fMRI studies in healthy subjects seem to further corroborate this functional interdependence by showing that intranasal trigeminal stimulation co-activates central olfactory areas such as the piriform cortex, while cutaneous trigeminal stimulation solely activates the traditional trigeminal areas $[1,19]$. The other way around, a very recent study in rats also suggests that gustatory function influences olfactory processing, thereby giving further evidence to the hypothesis of mutual chemosensory interaction [11].

The interactions within the chemical senses in people with congenital, compared to acquired, loss are less clear. First, congenital trigeminal and total gustatory losses are clinically extremely rare and poorly investigated. Second, for congenital anosmia, very few studies exist. One recently conducted study suggested that patients with congenital anosmia do not show any altered trigeminal function compared to patients who had acquired olfactory impairment [12]. The sparse literature on gustatory function in congenital anosmia does not overcome the level of case reports with contradictory results $[13,15]$. The number of patients in our study with congenital anosmia is too small $(n=2)$ to clarify this situation and, although both of them had taste strip values in the best third of the normal range, we think the issue of taste function in congenital anosmia must be addressed in future by investigating a larger sample size.

In a previous report, Vennemann et al. [33] tested olfactory and gustatory function in over a thousand patients without finding an association between taste and smell impairment. These authors used very rough taste testing, which could be a reason why their findings contrast with the present data. It further underlines that the gustatory decrease in olfactory impairment patients is of significant but moderate and subclinical extent and needs a sensible taste testing device to be revealed.

The authors of the previous studies identifying chemosensory interaction in health and disease have proposed different anatomical sites where this mutual modulation among senses could take place $[4,7,17]$. Based on the present clinical data, it remains purely speculative to propose a mechanism or site for this gustatory decrease after acquired olfactory impairment. Nevertheless, according to the abovementioned anatomical particularities of the chemical senses, we might remember that olfactory and trigeminal fibers, like taste and trigeminal fibers, are intermingled at a peripheral level. This has not yet been described for taste and olfactory fibers, with the nose having no gustatory innervation and the tongue no olfactory innervation. Solitary chemosensory single cells carrying bitter receptors have been observed within the nasal mucosa, but they seem to be related to the trigeminal rather than the taste system [10, 14]. Consequently, one could imagine that the decrease in gustatory function after acquired olfactory impairment observed here is due to central nervous changes. Thus, the most likely candidate regions are the amygdale, the thalamus, the insula or the orbitofrontal cortex [27]. As stroke patients with lesions of the orbitofronal cortex have been shown to exhibit more frequent and more pronounced taste alterations, even when they are unaware of that taste impairment, than patients with stoke lesions elsewhere [16], we dare to speculate that the presently observed interaction between the olfactory and gustatory functions could be due to neuronal modifications at the level of the orbitofrontal cortex. PET imaging studies investigating activation after smell, taste and mixed stimulation revealed that bimodal (smell and taste together) stimulation leads to an activation that is more than simply the sum of its parts [31]. These authors also found the orbitofrontal cortex to be the main region of interest for the observed smell-taste interaction.

Taken together, we provide further evidence that suggests that the hypotheses according to which chemical senses intimately interact could be extended to smell and taste, and that this is also reflected in patients with chemosensory dysfunction. It further raises the question whether chemical senses in general decrease mutually after acquired damage. The present findings also suggest that chemical senses, in contrast to other sensory modalities, do not tend to show compensatory mechanisms, but rather mutual weakening. However, it has to be kept in mind that the present data are from clinical observation and not a 
proof of any underlying mechanism. Psychophysical test results can be influenced by many co-factors, of which the most important are age, disease state and cognitive function. We could rule out age as a co-factor but we did not test for cognitive function and disease state, which is a shortcoming of the present data.

Besides bringing to light further evidence for the mutual chemosensory interaction hypothesis, these findings have a direct clinical impact on smell and taste in the impaired patient's workup in medico-legal issues. It might also explain a clinical observation that many patients with olfactory impairment report changes in food habits and particularly enhanced seasoning of foods. Taken together, the present findings further underline the role of chemosensory interaction and we feel that routine chemosensory investigation should include assessment of all chemical senses.

Acknowledgments We would like to thank Gerd Kobal, Stefan Kallert and Christian Mueller for their helpful advice concerning production and use of the "Taste Strips". The study was supported by a Grant of the Swiss National Fund for Scientific Research [SSMBS grant no. PASMA-119579/1] to BNL. Parts of this work have been published previously as Abstract (see Hummel et al., Chemical Senses 2001, 26, 1118).

\section{References}

1. Albrecht J, Kopietz R, Frasnelli J, Wiesmann M, Hummel T, Lundstrom JN (2010) The neuronal correlates of intranasal trigeminal function - an ALE meta-analysis of human functional brain imaging data. Brain Res Rev 62(2):183-196

2. Buchel C, Price C, Frackowiak RS, Friston K (1998) Different activation patterns in the visual cortex of late and congenitally blind subjects. Brain 121(Pt 3):409-419

3. Cain WS (1974) Contribution of the trigeminal nerve to perceived odor magnitude. Ann N Y Acad Sci 237:28-34

4. Cain WS, Murphy CL (1980) Interaction between chemoreceptive modalities of odour and irritation. Nature 284:255-257

5. Collignon O, Voss P, Lassonde M, Lepore F (2009) Cross-modal plasticity for the spatial processing of sounds in visually deprived subjects. Exp Brain Res 192:343-358

6. Cruz A, Green BG (2000) Thermal stimulation of taste. Nature 403:889-892

7. Dalton P, Doolittle N, Nagata H, Breslin PA (2000) The merging of the senses: integration of subthreshold taste and smell. Nat Neurosci 3:431-432

8. Doty RL, Bartoshuk LM, Snow JBJ (1991) Causes of olfactory and gustatory disorders. In: Getchell TV, Doty RL, Bartoshuk LM, Snow JBJ (eds) Smell and taste in health and disease. Raven Press, New York, pp 449-462

9. Doty RL, Shah M, Bromley SM (2008) Drug-induced taste disorders. Drug Saf 31:199-215

10. Finger TE, Bottger B, Hansen A, Anderson KT, Alimohammadi H, Silver WL (2003) Solitary chemoreceptor cells in the nasal cavity serve as sentinels of respiration. Proc Natl Acad Sci USA 100:8981-8986

11. Fortis-Santiago Y, Rodwin BA, Neseliler S, Piette CE, Katz DB (2010) State dependence of olfactory perception as a function of taste cortical inactivation. Nat Neurosci 13:158-159
12. Frasnelli J, Schuster B, Hummel T (2007) Subjects with congenital anosmia have larger peripheral but similar central trigeminal responses. Cereb Cortex 17:370-377

13. Gilliland AR (1921) The taste sensitivity of an anosmic subject. J Exp Psychol 4:318-326

14. Gulbransen B, Silver W, Finger TE (2008) Solitary chemoreceptor cell survival is independent of intact trigeminal innervation. J Comp Neurol 508:62-71

15. Hasan KS, Reddy SS, Barsony N (2007) Taste perception in Kallmann syndrome, a model of congenital anosmia. Endocr Pract 13:716-720

16. Heckmann JG, Stossel C, Lang CJ, Neundorfer B, Tomandl B, Hummel T (2005) Taste disorders in acute stroke: a prospective observational study on taste disorders in 102 stroke patients. Stroke 36:1690-1694

17. Hummel T, Barz S, Lotsch J, Roscher S, Kettenmann B, Kobal G (1996) Loss of olfactory function leads to a decrease of trigeminal sensitivity. Chem Senses 21:75-79

18. Hummel T, Kobal G, Gudziol H, Mackay-Sim A (2007) Normative data for the "Sniffin' Sticks" including tests of odor identification, odor discrimination, and olfactory thresholds: an upgrade based on a group of more than 3,000 subjects. Eur Arch Otorhinolaryngol 264:237-243

19. Iannilli E, Del Gratta C, Gerber JC, Romani GL, Hummel T (2008) Trigeminal activation using chemical, electrical, and mechanical stimuli. Pain 139:376-388

20. Kobal G, Hummel C (1988) Cerebral chemosensory evoked potentials elicited by chemical stimulation of the human olfactory and respiratory nasal mucosa. Electroencephalogr Clin Neurophysiol 71:241-250

21. Kobal G, Klimek L, Wolfensberger M, Gudziol H, Temmel A, Owen CM, Seeber H, Pauli E, Hummel T (2000) Multicenter investigation of 1,036 subjects using a standardized method for the assessment of olfactory function combining tests of odor identification, odor discrimination, and olfactory thresholds. Eur Arch Otorhinolaryngol 257:205-211

22. Landis BN, Hummel T, Lacroix JS (2005) Basic and clinical aspects of olfaction. Adv Tech Stand Neurosurg 30:69-105

23. Landis BN, Welge-Luessen A, Bramerson A, Bende M, Mueller CA, Nordin S, Hummel T (2009) "Taste Strips"-a rapid, lateralized, gustatory bedside identification test based on impregnated filter papers. J Neurol 256:242-248

24. Merabet LB, Hamilton R, Schlaug G, Swisher JD, Kiriakopoulos ET, Pitskel NB, Kauffman T, Pascual-Leone A (2008) Rapid and reversible recruitment of early visual cortex for touch. PLoS One 3:e3046

25. Mueller C, Kallert S, Renner B, Stiassny K, Temmel AF, Hummel T, Kobal G (2003) Quantitative assessment of gustatory function in a clinical context using impregnated "taste strips". Rhinology 41:2-6

26. Pfaar O, Landis BN, Frasnelli J, Huttenbrink KB, Hummel T (2006) Mechanical obstruction of the olfactory cleft reveals differences between orthonasal and retronasal olfactory functions. Chem Senses 31:27-31

27. Rolls ET (2005) Taste, olfactory, and food texture processing in the brain, and the control of food intake. Physiol Behav 85:4556

28. Rolls ET, Baylis LL (1994) Gustatory, olfactory, and visual convergence within the primate orbitofrontal cortex. J Neurosci 14:5437-5452

29. Schaefer ML, Bottger B, Silver WL, Finger TE (2002) Trigeminal collaterals in the nasal epithelium and olfactory bulb: a potential route for direct modulation of olfactory information by trigeminal stimuli. J Comp Neurol 444:221-226

30. Shepherd GM (2006) Smell images and the flavour system in the human brain. Nature 444:316-321 
31. Small DM, Jones-Gotman M, Zatorre RJ, Petrides M, Evans AC (1997) Flavor processing: more than the sum of its parts. Neuroreport 8:3913-3917

32. Todrank J, Bartoshuk LM (1991) A taste illusion: taste sensation localized by touch. Physiol Behav 50:1027-1031

33. Vennemann MM, Hummel T, Berger K (2008) The association between smoking and smell and taste impairment in the general population. J Neurol 255:1121-1126
34. Voss P, Lassonde M, Gougoux F, Fortin M, Guillemot JP, Lepore F (2004) Early- and late-onset blind individuals show supranormal auditory abilities in far-space. Curr Biol 14:1734-1738

35. Whitehead MC, Beeman CS, Kinsella BA (1985) Distribution of taste and general sensory nerve endings in fungiform papillae of the hamster. Am J Anat 173:185-201 